\title{
Three-Dimensional Additively Manufactured Microstructures and Their Mechanical Properties
}

\author{
THERON M. RODGERS (i), ${ }^{1,3}$ HOJUN LIM, ${ }^{1}$ and JUDITH A. BROWN ${ }^{2}$ \\ 1.-Department of Computational Materials and Data Science, Sandia National Laboratories, \\ Albuquerque, NM, USA. 2.-Department of Fluid and Reactive Processes, Sandia National Lab- \\ oratories, Albuquerque, NM, USA. 3.-e-mail: trodger@sandia.gov
}

\begin{abstract}
Metal additive manufacturing (AM) allows for the freeform creation of complex parts. However, AM microstructures are highly sensitive to the process parameters used. Resulting microstructures vary significantly from typical metal alloys in grain morphology distributions, defect populations and crystallographic texture. AM microstructures are often anisotropic and possess three-dimensional features. These microstructural features determine the mechanical properties of AM parts. Here, we reproduce three "canonical" AM microstructures from the literature and investigate their mechanical responses. Stochastic volume elements are generated with a kinetic Monte Carlo process simulation. A crystal plasticity-finite element model is then used to simulate plastic deformation of the AM microstructures and a reference equiaxed microstructure. Results demonstrate that AM microstructures possess significant variability in strength and plastic anisotropy compared with conventional equiaxed microstructures.
\end{abstract}

\section{INTRODUCTION}

Additive manufacturing (AM) techniques are increasing in popularity as they enable building of complex three-dimensional parts through layer-bylayer synthesis. Typical metallic AM parts exhibit non-traditional microstructural features resulting from AM processes. These features include grain shape and size distributions, crystallographic texture, porosity, dendrite-scale solidification structures and compositional variation, all of which can significantly affect mechanical behavior. In this work, we focus on the grain morphology and crystallographic texture of AM materials and their effects on mechanical properties.

AM microstructures are inherently three-dimensional and require commensurate 3D simulation techniques to properly capture their morphologies and properties. The local microstructure within a heat source raster path is governed by solidification processes in the moving melt pool. However, the microstructure of a multilayer build is also influenced by the overlap of multiple scan tracks within a single layer and by partial or full remelting of previously deposited layers. ${ }^{1}$ In many AM studies, a microstructure with elongated columnar grains and a $\left\langle\begin{array}{llll}1 & 0 & 0\end{array}\right.$ fiber texture has been observed. ${ }^{2-9}$ This texture typically occurs in cubic materials, where the $\left\langle\begin{array}{lll}1 & 0 & 0\end{array}\right\rangle$ crystal direction is the preferred growth direction. ${ }^{0,11}$ These microstructures have been observed with long vector scanning strategies and high beam power densities. ${ }^{1,2,4}$ Examples of metal AM microstructures are shown in the top row of Fig. 1 along with an equiaxed microstructure commonly observed in wrought metal. Columnar, fibertextured microstructures (where one crystallographic direction is preferentially aligned with a reference frame direction, as shown in Fig. 1c) can occur with varying texture strengths., ${ }^{1,2}, 8$

The $\left\langle\begin{array}{llll}1 & 0 & 0\end{array}\right\rangle$ crystal direction can also be aligned at various angles with respect to the build and scan vectors of the AM part. For example, Wei et al. ${ }^{5}$ reported a uniform $\left\langle\begin{array}{llll}1 & 0 & 0\end{array}\right\rangle$ texture oriented at an angle of $60^{\circ}$ with the scan direction for unidirectional scanning and for bidirectional scanning a $\left\langle\begin{array}{lll}1 & 0 & 0\end{array}\right)$ texture that started at $45^{\circ}$ with the scan direction in the first layer and then alternated the direction of the primary dendrites by $90^{\circ}$ between each layer. In some instances, a $\left\langle\begin{array}{llll}1 & 0 & 0\end{array}\right\rangle$ cube texture has been reported rather than a fiber texture. ${ }^{1,6}$ Differing mechanical properties have been observed in AM parts with strong fiber $\left\langle\begin{array}{llll}1 & 0 & 0\end{array}\right\rangle$ textured microstructures 
(a) Equiaxed

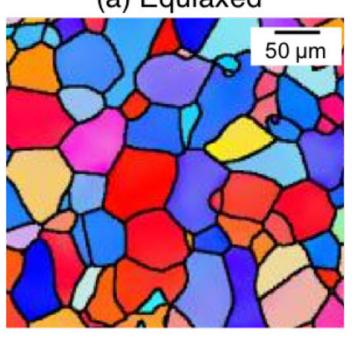

Microtexture in alternating layers
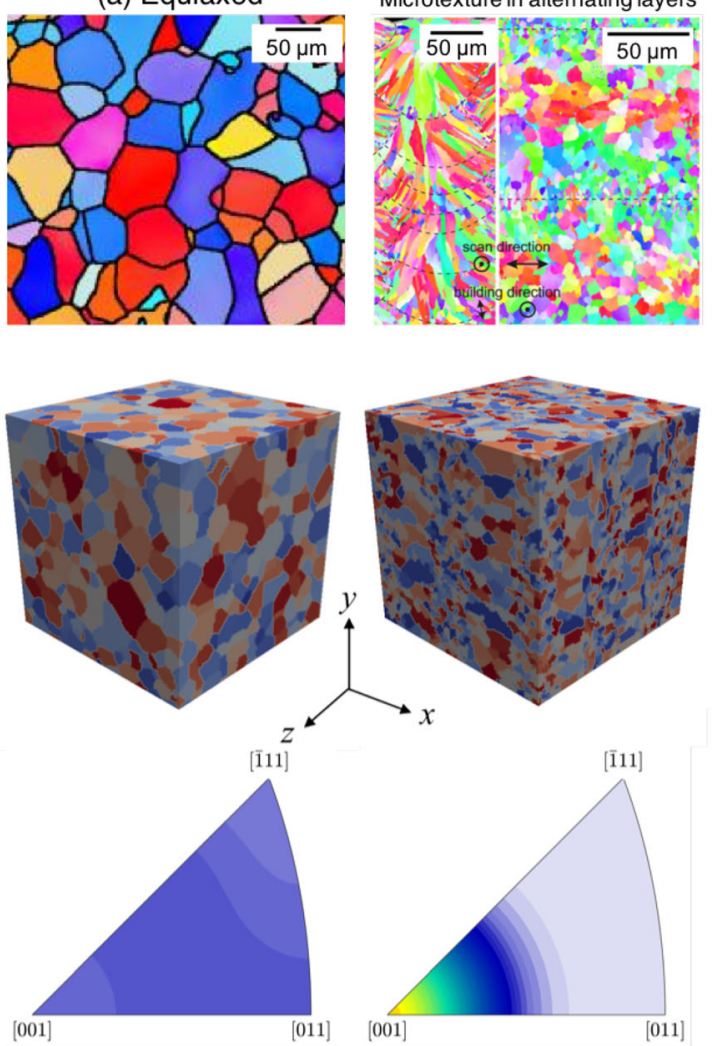

(c) AM2

$<100>$ fiber texture
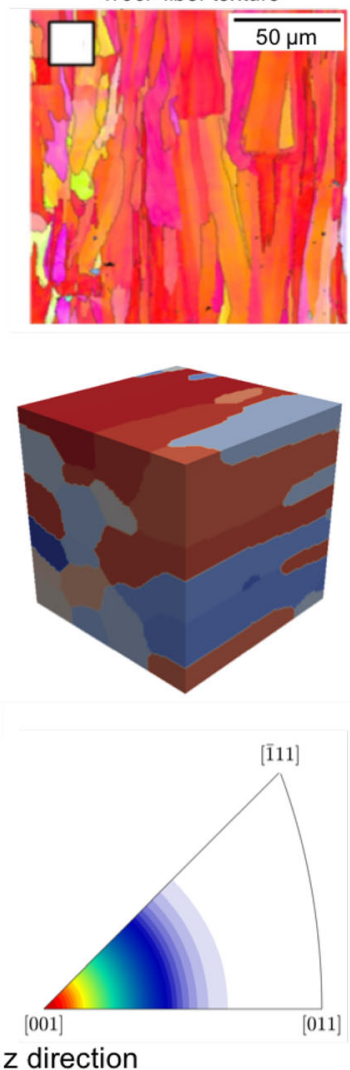

(d) $\mathrm{AM} 3$

No macroscale texture
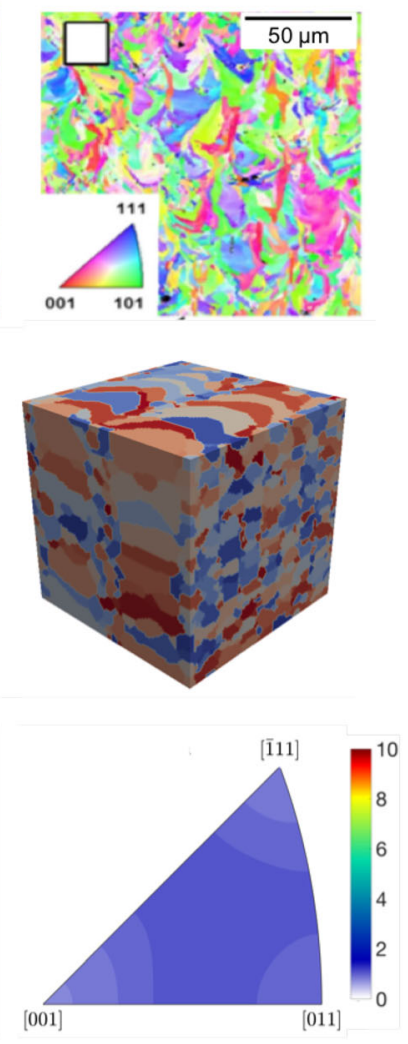

Fig. 1. Top row: Experimental EBSD images of representative microstructures. AM1 image re-used with permission from Ref. 1. AM2 and AM3 images re-used with permission from Ref. 2. Middle row: 3D renderings of synthetic microstructures (colors represent grain IDs). Bottom row: $Z$ direction inverse pole figures representing each synthetic microstructure's initial crystallographic texture.

(Fig. 1c), with a decrease of Young's modulus compared with non-textured AM microstructures (Fig. 1d). ${ }^{2}$ Additionally, elastic properties of AM parts can vary significantly depending on the orientation of the build direction with respect to the loading direction. ${ }^{12}$ AM materials with strong texture are also shown to have anisotropy in failure processes, such as fatigue crack growth. ${ }^{3}$

AM microstructures that do not feature multilayer columnar grains and strong textures often possess grains that are elongated in the direction of the solid/liquid interface and local thermal gradient during solidification. Thus, the impact of the beam scan pattern used during manufacturing is exhibited in the microstructure. ${ }^{13-15}$ An example of this untextured microstructure is shown in Fig. 1d for $316 \mathrm{~L}$ stainless steel. ${ }^{2}$ Note that the morphology shown in Fig. 1d is still distinctly different from wrought material, with elongated grains and evidence of melt pool boundaries. These finer, more isotropic microstructures are generally produced by lower power densities and shorter vector scan strategies that change directions, such as island scanning. ${ }^{1,16,17}$ Even if the microstructure as a whole does not have a significant global texture, local microtextures can still be present because of the cyclic thermal history seen during multilayer builds. Thijs et al. studied several different scan patterns and found repeated localized bands of elongated grains with $\left\langle\begin{array}{lll}1 & 0 & 0\end{array}\right\rangle$ texture when a global texture was not present (Fig. 1b). ${ }^{1}$

There has been significant previous work toward determining the mechanical behavior of AM metals, including the use of crystal plasticity simulations to model AM microstructures. However, few computational studies have utilized realistic, three-dimensional microstructures created by process simulations. Several studies have utilized two-dimensional experimental or idealized microstructures. ${ }^{18,19}$ Others have used idealized threedimensional microstructures representing AM grain aspect ratios and crystallographic textures. ${ }^{20,21}$ Recently, Herriott et al. used a 3D Cellular Automata process model and crystal plasticity simulations to simulate the spatial variation of microstructure and mechanical properties within a rectangular AM build. The study also varied the nucleation parameter used in the AM process simulation to study the effect of grain size on response. ${ }^{22}$ Several experimental investigations have also studied variation within an AM build..$^{23-26}$ The study presented in this article focuses on microstructure and property variation between disparate AM processes rather than spatial variation within a single build. 
In this article, we evaluate the influence of process-induced variation in AM microstructures using a fully computational approach. First, stochastic volume elements with synthetic microstructures representative of experimental literature examples were generated with $\mathrm{kMC}$ AM process simulations. Crystallographic orientations were then mapped to the SVE from experimentally inspired orientation distribution functions. Finally, mechanical responses were investigated with crystal plasticity-finite element method (CP-FEM) simulations.

\section{METHODS}

The "app_additive" module in the Stochastic Parallel PARticle Kinetic Simulator (SPPARKS) simulation suite developed at Sandia National Laboratories was used to generate the synthetic microstructures. The process simulations explicitly simulate the traversal of a molten zone through the simulation domain as occurs in additive processes. Many process parameters can be varied including the molten pool shape, raster pattern, layer height and temperature gradient around the molten zone. The method has been described extensively in previous publications, and the reader is referred to these works for further details. ${ }^{13,27-29}$

After the generation of stochastic volume elements (SVEs) with AM microstructures in SPPARKS, the SVEs were assigned a crystallographic texture. First, the "uniformODF" and "fibreODF" functions of the MTEX MATLAB toolbox ${ }^{30}$ were used to generate uniformly random and fiber orientation distribution functions. Individual grain orientations were sampled from the distributions using the "calcOrientations" function. Next, a Python script was used to associate each grain ID in the synthetic microstructures to an orientation from the MTEX distributions. For fiber-textured and untextured microstructures, orientations were selected from the fiber and uniformly random distributions, respectively. For microstructures with no global texture but layer-wise microtextures, orientations were selected from both distributions. Grains $>100 \mu \mathrm{m}^{3}$ were assigned orientations from the fiber distribution, while smaller grains received orientations from the uniformly random distribution. The MTEX and Python scripts are included as supplemental material.

AM structures generated from SPPARKS were then converted to hexahedral finite element meshes for mechanical simulations using CP-FEM analysis. A well-established rate-dependent crystal plasticity framework implemented in a three-dimensional Sandia code for finite element analysis of solids and structures (JAS3D) was used. ${ }^{31-34}$ A simple power-law function is used to represent slip rates: ${ }^{35}$

$$
\dot{\gamma}^{\alpha}=\dot{\gamma}_{0}^{\alpha}\left(\frac{\tau^{\alpha}}{g^{\alpha}+g_{d}^{\alpha}}\right)^{1 / m}
$$

where $\dot{\gamma}_{0}^{\alpha}$ is the reference shear rate, $m$ is the rate sensitivity factor, $\tau^{\alpha}$ is the resolved shear stress, $g^{\alpha}$ is the slip resistance, and $g_{d}^{\alpha}$ is the grain size strengthening. Here, $g^{\alpha}$ is represented by a slipbased hardening as follows:

$$
\dot{g}^{\alpha}=\sum_{\beta}^{12} h^{\alpha \beta}\left|\dot{\gamma}^{\alpha}\right|
$$

The hardening coefficient, $h^{\alpha \beta}$, is represented as: ${ }^{36}$

$$
h^{\alpha \beta}=\mathbf{q}^{\alpha \beta} h_{0}\left(1-\frac{g^{\beta}}{g_{s}}\right)^{a}
$$

where $\mathbf{q}^{\alpha \beta}$ is the $12 \times 12$ hardening matrix, $h_{0}$ is the initial hardening rate, $g_{s}$ is the saturated flow stress, and $a$ is the hardening exponent. The diagonal $\left(q_{s}\right)$ and off-diagonal $\left(q_{l}\right)$ terms in $\mathbf{q}^{\alpha \beta}$ determine the self and latent hardening effects. The grain size contribution, $g_{d}^{\alpha}$, is represented by the Hall-Petch relation, $k d^{-1 / 2}$, where $k$ is the HallPetch slope and $d$ is the equivalent spherical grain diameter. The Hall-Petch relationship used here accounts for grain size-dependent hardening, but does not account for anisotropic grain shapes. Material parameters in Eqs. 1-3 were fit to the typical stress-strain response of $316 \mathrm{~L}$ stainless steel. Table I lists material parameters used in CP-FEM simulations.

We generated 20 realizations of $1 \mathrm{~mm}^{3}$ stochastic volume elements (SVEs) for each microstructure class. Using this approach, the largest standard deviation for any response value for a microstructure class was $\approx 5 \%$.

\section{RESULTS}

\section{Microstructure Generation}

SVEs representing three microstructure classes from the literature were generated using the procedure described in the "Methods" section and are shown in the center row of Fig. 1. The SPPARKS input file used to generate each synthetic microstructure is included in the supplemental material. The random number generator's seed value was varied to generate the 20 realizations of each microstructure class. 'AM1' is a bimodal structure. The microstructure is composed of large and small grains whose size and crystallographic orientation depend upon their location within the scan pattern. The microstructure alternates between larger grains with their $\left[\begin{array}{lll}0 & 0 & 1\end{array}\right]$ direction aligned with the $z$ direction (build direction) and smaller grains with random orientations. This can be observed in the inverse pole figure (IPF) of Fig. 1b, which shows preferred orientations near the $\left[\begin{array}{lll}0 & 0 & 1\end{array}\right]$ pole. 'AM2' is a columnar microstructure with a strong [ $\left[\begin{array}{lll}0 & 0 & 1\end{array}\right]$ fiber texture. Columnar grains and the fiber texture are both aligned with the $z$ direction. The 'AM3' microstructure contains grains that are uniform in size through the domain and a 
uniformly random crystallographic texture. The grains are elongated along the local solidification direction and have a periodically repeating pattern imposed by the molten pool's raster.

\section{Variation in Uniaxial Loading Response}

To understand the microstructure-mechanical property relationships in AM materials, each microstructure was deformed up to $10 \%$ strain (well within the uniform strain regime of $316 \mathrm{~L}$ ) along the $z$ direction (build direction). Simulated maps of local von Mises stress (top row) and equivalent plastic strain (EQPS, bottom row) fields are shown in Fig. 2. Global stress-strain responses from all 80 SVEs are shown in Fig. 3a. Significant variation is observed in both the elastic and plastic regimes of the stress-strain curves. The maximum yield stress variation between the structures was $\approx 60 \mathrm{MPa}$. AM2 (the columnar structure) was the softest, while the equiaxed and smaller-grained AM structures (AM1, AM3) were stronger. This is consistent with experimental observations of microstructure-dependent strength in AM material. ${ }^{2,37}$ The microstructure classes also exhibited differing variability in mechanical response. Equiaxed and AM1 SVEs demonstrated minimal variation, while AM2 and AM3 SVEs demonstrated larger deviations. The smaller variation in equiaxed and AM1 SVEs is attributed to the presence of many small grains that reduce the overall variability of the SVE response.

Local mechanical responses can be quantified by studying the von Mises stress throughout the domain. Figure $3 \mathrm{~b}$ shows the distribution of perelement von Mises stresses for the four microstructures at $10 \%$ strain. AM1 microstructures possessed a significant fraction of grains whose $\left[\begin{array}{lll}0 & 0 & 1\end{array}\right]$ directions aligned with the loading direction as indicated by the distribution's maxima near the low value of $350 \mathrm{MPa}$. The bimodal microstructure in AM1 is apparent from a smaller, secondary peak near $575 \mathrm{MPa}$. In the columnar AM2 microstructures, a large portion of the simulation domain is filled by large oriented grains, demonstrated by the large peak near $325 \mathrm{MPa}$. For the equiaxed and AM3 microstructures, the distribution of stress is more uniform. There are concentrations of grains with a von Mises stress between 500-600 MPa, but the peaks are much broader than those of AM1 and AM2.

\section{Plastic Anisotropy}

The stress-strain curves in Fig. 3a demonstrate microstructure-induced material variability. In addition to material variability along each loading direction, different magnitudes of plastic anisotropy are expected for the different AM microstructures considered in this work. AM2 is expected to display

Table I. Material parameters used in CP-FEM simulations

\begin{tabular}{|c|c|c|c|c|c|c|c|}
\hline Param. & Values & Param. & Values & Param. & Values & Param. & Values (GPa) \\
\hline$m$ & 0.012 & $h_{0}$ & $300 \mathrm{MPa}$ & $q_{s}$ & 1.0 & $C_{11}$ & 204.6 \\
\hline$\dot{\gamma}_{0}$ & $1 \mathrm{~s}^{-1}$ & $g_{s}$ & $380 \mathrm{MPa}$ & $q_{l}$ & 1.4 & $C_{12}$ & 137.7 \\
\hline$g_{0}$ & $75 \mathrm{MPa}$ & $a$ & 2.5 & $k$ & $0.5455 \mathrm{MN} / \mathrm{m}^{1 / 2}$ & $C_{44}$ & 126.2 \\
\hline
\end{tabular}
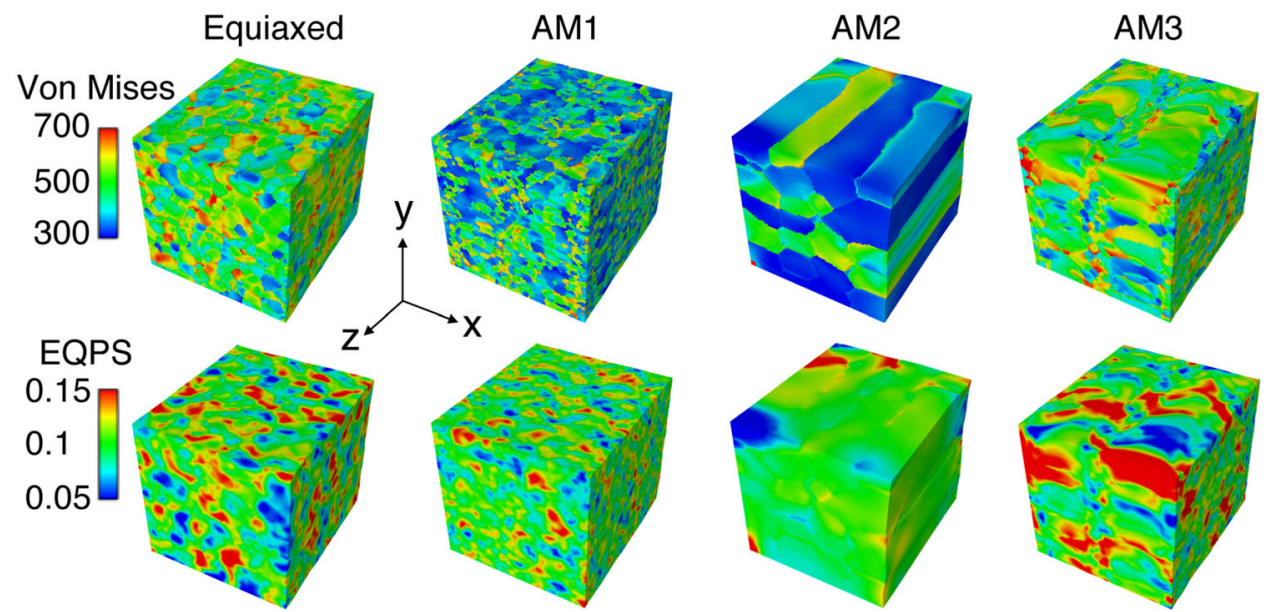

Fig. 2. Microstructures showing von Mises stress (top) and equivalent plastic strain (bottom) for equiaxed, AM1 (layered), AM2 (columnar) and AM3 (uniform) microstructures at $10 \%$ strain. 
(a) Stress-strain response to Z-loading

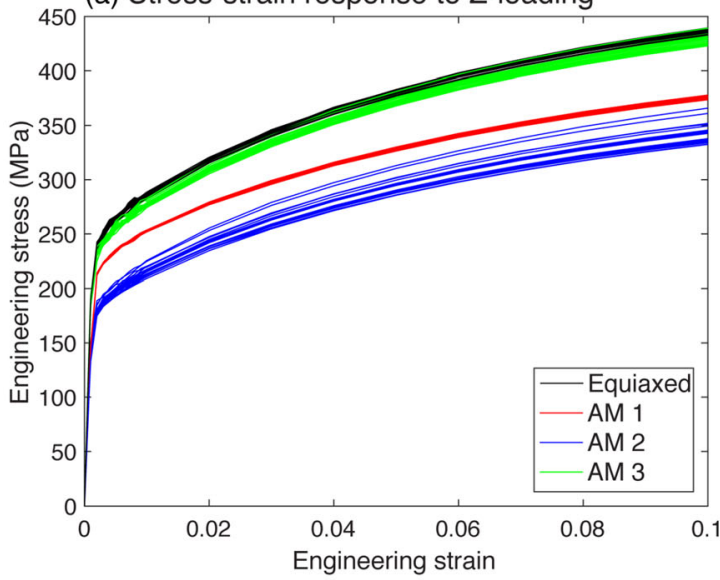

(b) Distribution of von Mises stress at $10 \%$ strain

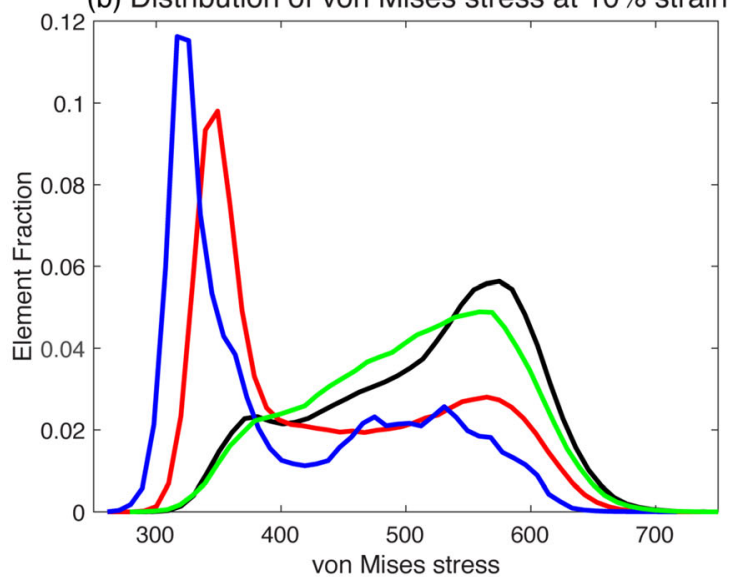

Fig. 3. (a) Global stress-strain response curve for each microstructure SVE. Load was applied parallel to the $z$ direction. (b) Distribution of von Mises stress at $10 \%$ strain. The $y$ axis shows the fraction of finite elements with a given von Mises value. The AM1 microstructure contained a significant number of grains with their $\left[\begin{array}{lll}0 & 0 & 1\end{array}\right]$ directions aligned with the loading direction, resulting in a large peak at low von Mises. AM2 possessed large, $\left[\begin{array}{lll}0 & 0 & 1\end{array}\right]$-oriented grains as shown by the large peak at low von Mises values. Equiaxed and AM3 microstructures demonstrated more uniform distributions of stress with maximums at higher values.

the highest degree of anisotropy because of both the grain morphology and its strong crystallographic texture. To quantify these plastic anisotropies, CP simulations were repeated with the load applied to the $x$ and $y$ directions. The stress-strain responses for the three loading conditions are shown in Fig. 4. Each line represents the mean response of 20 microstructures and is enveloped by the standard deviation. Variation in elastic response was determined by calculating Young's modulus, which is shown in Table II for each simulation. Directional variation of Young's modulus was small for each microstructure class (consistent with experiments on $304 \mathrm{~L}^{38}$ ), but the equiaxed and AM3 results had moduli $\approx 50 \mathrm{GPa}$ higher than those of AM1 and AM2.

As expected, AM2 displayed the largest magnitude of anisotropy for both yield stress and flow stresses at 5 and $10 \%$ deformations. The average yield stress (measured at $2 \%$ deviation from linear response) and its standard deviation for each microstructure and loading condition are listed in Table II. As apparent in Fig. 4, the equiaxed and AM3 microstructures possessed the highest yield stresses for all loading directions. The equiaxed microstructure demonstrated negligible plastic anisotropy having $<1 \mathrm{MPa}$ of variation in yield stress. AM1 was significantly weaker, but displayed a similar lack of anisotropy; its yield stress also varied by $<1 \mathrm{MPa}$ with loading direction. This resulted from the presence of both large soft grains (which reduced the yield stress) and many small grains (which reduced the variation in response). AM2 possessed both significantly lower yield stress and the highest degree of plastic anisotropy with 7\% variation between the highest and lowest values. The strong plastic anisotropy in AM2 is consistent with the Hall-Petch relationship found in both experimental observations ${ }^{2}$ and other modeling efforts. ${ }^{39}$ As noted by Liu et al., ${ }^{39}$ the effective grain boundary spacing (i.e., effective grain size) varies with respect to different loading directions.

The standard deviations of the yield stress shown in Table II were found to vary with average grain size. AM2 demonstrated the largest amount of deviation with a value $>4 \mathrm{MPa}$ for all loading directions. These microstructures had the fewest distinct grains, which increased variation in the SVE's response. The lowest standard deviation was observed in the AM1 microstructure, which included a large number of small grains that reduced variation. The AM3 and equiaxed grain sizes (and the stress deviations) fell between these two extremes.

Values of engineering stress at 5\% and $10 \%$ strain along different loading directions are also shown in Table II. For AM2, standard deviations in flow stresses increased with increasing strain, although the standard deviation as a percentage of stress remained relatively constant near $3 \%$. For the other microstructures, the standard deviation only slightly increased, resulting in a decreasing standard deviation as a percentage of the stress response.

\section{DISCUSSION}

Although a direct comparison with experimental results was beyond the scope of the current study, comparisons can be made with trends observed in experimental studies. The AM2 and AM3 microstructures studied here were respectively representative of the $1000 \mathrm{~W}$ and $400 \mathrm{~W}$ experimental structures in Ref. 2, which also reports tensile tests of the two as-built conditions. In Ref. 2 , the ratio between $400 \mathrm{~W}$ and $1000 \mathrm{~W}$ microstructures' yield stresses was $\approx 1.2$, while the equivalent $(z$ - 

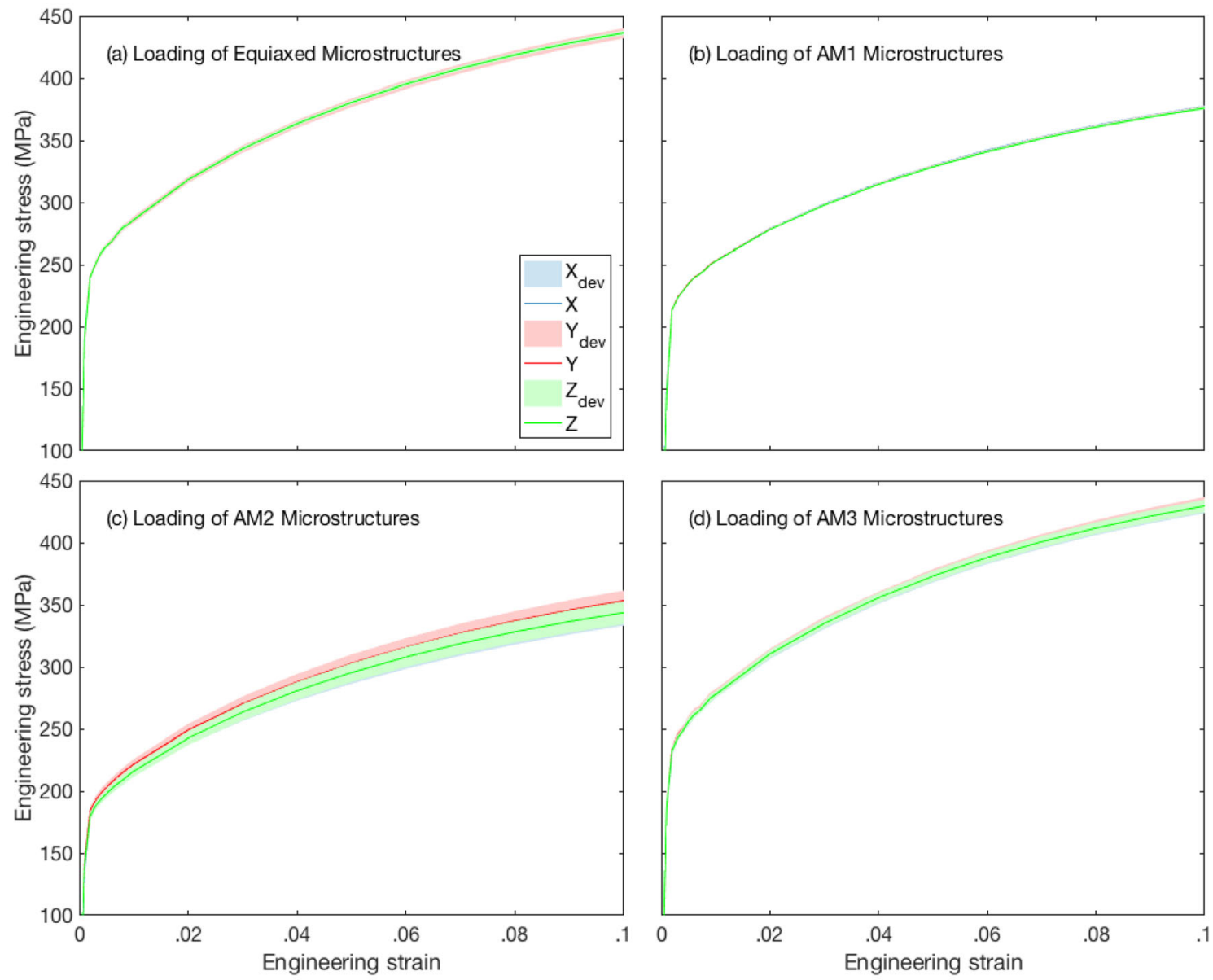

Fig. 4. Mechanical responses of the synthetic microstructures in three orthogonal loading directions. Each line represents the mean response of 20 microstructures and is enveloped by a shaded region indicating the standard deviation.

Table II. Values of Young's modulus, yield stress and engineering stress at 5\% and 10\% elongation

\begin{tabular}{lcccc}
\hline Load direction & Equiaxed & AM1 & AM2 & AM3 \\
\hline Young's modulus $(\mathrm{GPa})$ & & & & \\
$X$ & $195.30(1.20)$ & $149.53(0.81)$ & $141.15(7.75)$ & $194.04(4.11)$ \\
$Y$ & $194.33(2.81)$ & $147.88(0.84)$ & $146.86(6.28)$ & $193.01(3.50)$ \\
$Z$ & $194.83(1.32)$ & $147.69(0.81)$ & $147.07(7.44)$ & $194.59(3.91)$ \\
Yield stress (MPa) & & & & \\
$X$ & $191.15(1.33)$ & $151.61(0.61)$ & $135.91(4.97)$ & $184.77(4.08)$ \\
$Y$ & $190.51(2.44)$ & $150.77(0.68)$ & $138.40(7.55)$ & $185.46(2.56)$ \\
$Z$ & $190.93(1.23)$ & $151.61(0.68)$ & $128.81(4.12)$ & $181.86(3.06)$ \\
Engineering stress at 5\% (MP) & & & \\
$X$ & $381.33(1.43)$ & $329.64(0.76)$ & $294.86(8.50)$ & $372.71(4.61)$ \\
$Y$ & $380.28(3.48)$ & $328.93(1.08)$ & $303.03(6.98)$ & $374.78(3.90)$ \\
$Z$ & $380.61(1.59)$ & $328.54(1.01)$ & $295.39(7.62)$ & $373.06(4.12)$ \\
Engineering stress at $10 \%(\mathrm{MPa})$ & & & \\
$X$ & $437.27(1.62)$ & $377.28(0.91)$ & $343.31(10.05)$ & $429.15(5.47)$ \\
$Y$ & $436.14(4.12)$ & $376.43(1.23)$ & $353.48(8.10)$ & $432.16(4.63)$ \\
$Z$ & $436.51(1.85)$ & $376.06(1.13)$ & $343.77(9.28)$ & $429.59(4.96)$ \\
\hline
\end{tabular}

Average values from 20 simulations are shown with the corresponding standard deviation in parentheses. 
direction) simulated AM3 and AM2 yield stresses determined here had a ratio of 1.4. The ratio of Young's modulus displayed a similar trend, as the experimental results showed a ratio of $\approx 1.5$, and the simulation results varied by a ratio of 1.32 . Thus, the trends demonstrated by the simulations are consistent with the experimental results.

Recent experiments have shown that AM 316L stainless steel produced with an optimized process (resulting in few defects) can possess both higher UTS and ductility than wrought. ${ }^{40,41}$ These behaviors have been attributed to microstructure features at the sub-grain scale, including solidification cellular/dendritic structures (and corresponding microsegregation), second phase particles, accumulated dislocation densities and nitrogen uptake. ${ }^{25}$ These strengthening features are not included in the current CP formulation and result in lower predicted mechanical properties than measured experimentally. The evolution of these fine strengthening features can be predicted with microscale simulations of solidification. However, the ability to simulate the formation of three-dimensional polycrystalline microstructures with these features is beyond current computational capabilities. Future microstructure simulation methods could incorporate a multiscale approach to simulate these subgrain structures within a polycrystalline domain.

Additional properties not captured in this work include surface roughness, residual stress and porosity, which are commonly present in as-built AM parts. Residual stress can be categorized into macroscopic (type I) and microscopic (types II and III) length scales. The simulation volumes used here are too small to capture macroscopic variation directly, but variation of microscopic residual stress could be studied through linkages with process simulations and/or inclusion of the fine-scale features discussed above in future crystal plasticity simulations. Internal porosity defects are often caused by complex phenomena such as keyholing, lack of fusion or trapped gasses within the powder feedstock. ${ }^{42}$ Although computationally intensive fluid dynamic simulations are required to capture these effects in full fidelity, simplified process models may be able to incorporate these defects in synthetic microstructures with reduced computational costs. An increasingly large body of experimental work has analyzed porosity distributions in $3 \mathrm{D}$ and could be mined to produce statistical distributions of porosity, which could then be imposed on synthetic microstructures. ${ }^{4-45}$ In addition to the grain morphology and crystal orientations studied here, such features (subgrain structures, dislocations, porosity, residual stresses) will govern local stress states and determine the fracture mechanisms of a particular AM material. Thus, it is essential to incorporate these aspects in future studies for the accurate simulation of fatigue and fracture properties. ${ }^{23,24}$
In this study, identically sized simulation volumes were used for each microstructural class. Due to the large variation in average grain size, each microstructural class had significantly different number of unique grains, which contributed to AM2 (with the largest and fewest grains) having a greater standard deviation in response and may have also limited the anisotropic response by limiting the length/radius ratio of the columnar grains. This demonstrates the challenge of determining a uniformly "large-enough" representative volume element for AM material. Columnar microstructures with grains that extend over hundreds of microns may not be representable by an RVE for feature sizes of interest. Future work will study the convergence behavior of these microstructures over increasing SVE sizes.

\section{CONCLUSION}

A Monte Carlo-based process simulation was used to generate a data set representing multiple instances of three commonly observed categories of three-dimensional AM microstructures and a baseline equiaxed microstructure. Twenty instantiations of each microstructural class were used in CP-FEM simulations. The simulations demonstrated that AM microstructures and crystallographic textures can have a substantial effect on mechanical response. The microstructures demonstrated varying elastic moduli, yield stresses and plastic response that corresponded well with the relative experimental values. The standard deviation of each response was also found to vary with microstructure properties. Additionally, anisotropic response was observed for the AM2 (columnar) microstructure, which is a common as-built microstructure in metal powder bed processes. To achieve more quantitative accuracy in CP-FEM simulations of AM materials, detailed microstructural features such as dendrite-scale solidification structures, defect densities and low-angle boundaries need to be considered. This work demonstrates an integrated computational materials engineering (ICME) approach that directly bridges process modeling to three-dimensional materials mechanics. This enables more efficient AM materials design and characterization in engineering applications.

\section{ACKNOWLEDGEMENTS}

The authors thank Jonathan Madison and Allen Roach for their helpful comments and discussion.

This paper describes objective technical results and analysis. Any subjective views or opinions that might be expressed in the paper do not necessarily represent the views of the US Department of Energy or the United States Government. Sandia National Laboratories is a multi-mission laboratory managed and operated by National Technology and Engineering Solutions of Sandia, LLC, a wholly owned subsidiary of Honeywell International, Inc., 
for the US Department of Energy's National Nuclear Security Administration under contract DENA0003525.

\section{OPEN ACCESS}

This article is distributed under the terms of the Creative Commons Attribution 4.0 International License (http://creativecommons.org/licenses/by/4.0/), which permits unrestricted use, distribution, and reproduction in any medium, provided you give appropriate credit to the original author(s) and the source, provide a link to the Creative Commons license, and indicate if changes were made.

\section{REFERENCES}

1. L. Thijs, K. Kempen, J.P. Kruth, and J. Van Humbeeck, Acta Mater. 61, 1809 (2013).

2. T. Niendorf, S. Leuders, A. Riemer, H.A. Richard, T. Troster, and D. Schwarze, Metall. Mater. Trans. B 44B, 794 (2013).

3. A. Riemer, S. Leuders, M. Thone, H. Richard, T. Troster, and T. Niendorf, Eng. Fract. Mech. 120, 15 (2014).

4. T. Niendorf, F. Brenne, and M. Schaper, Metall. Mater. Trans. B 45B, 1181 (2014).

5. H. Wei, J. Mazumder, and T. DebRoy, Sci. Rep. 5, 16446 (2015).

6. G. Dinda, A. Dasgupta, and J. Mazumder, Scr. Mater. 67, 503 (2012).

7. I. Yadroitsev, P. Krakhmalev, I. Yadroitsava, S. Johansson, and I. Smurov, J. Mater. Process. Technol. 213, 606 (2013).

8. K. Saeidi, X. Gao, Y. Zhong, and Z. Shen, Mater. Sci. Eng. A 625, 221 (2015).

9. P.A. Kobryn and S.L. Semiatin, JOM J. Miner. Met. Mater. Soc. 53(9), 40 (2001).

10. M. Glicksman, Principles of Solidification (Springer, New York, 2011).

11. S. Kou, Welding Metallurgy, 2nd edn. (John Wiley and Sons, New Jersey, 2003).

12. L. Hitzler, J. Hirsch, B. Heine, M. Merkel, W. Hall, and A. Ochsner, Materials 10, 1136 (2017).

13. T.M. Rodgers, J.D. Madison, and V. Tikare, Comput. Mater. Sci. 135, 78 (2017).

14. A.T. Polonsky, M.P. Echlin, W.C. Lenthe, R.R. Dehoff, M.M. Kirka, and T.M. Pollock, Mater. Charact. 143, 171 (2018).

15. A.T. Polonsky, C.A. Lang, K.G. Kvilekval, M.I. Latypov, M.P. Echlin, B.S. Manjunath, and T.M. Pollock, Integr. Mater. Manuf. Innov. 8, 37-51 (2019).

16. R. Casati, J. Lemke, and M. Vedani, J. Mater. Sci. Technol. 32,738 (2016).

17. J. Suryawanshi, K. Prashanth, and U. Ramamurty, Mater. Sci. Eng. A 696, 113 (2017).

18. M.B. Gorji, T. Tancogne-Dejean, and D. Mohr, Acta Mater. 148, 442 (2018).

19. K. Kapoor, Y.S.J. Yoo, T.A. Book, J.P. Kacher, and M.D. Sangid, J. Mech. Phys. Solids 121, 447 (2018).

20. W. Yan, S. Lin, O.L. Kafka, C. Yu, Z. Liu, Y. Lian, S. Wolff, J. Cao, G.J. Wagner, and W.K. Liu, Front. Mech. Eng. 13(4), 482 (2018).

21. A. Ahmadi, R. Mirzaeifar, N.S. Moghaddam, A.S. Turabi, H.E. Karaca, and M. Elahinia, Mater. Des. 112, 328 (2016).
22. C. Herriott, X. Li, N. Kouraytem, V. Tari, W. Tan, B. Anglin, A.D. Rollett, and A.D. Spear, Modell. Simul. Mater. Sci. Eng. 27(2), 025009 (2019).

23. J.J. Lewandowski and M. Seifi, Annu. Rev. Mater. Res. 46(1), 151 (2016).

24. M. Seifi, A. Salem, D. Satko, J. Shaffer, and J.J. Lewandowski, Int. J. Fatigue 94, 263 (2017).

25. T.R. Smith, J.D. Sugar, C.S. Marchi, and J.M. Schoenung, Acta Mater. 164, 728 (2019).

26. K.L. Johnson, T.M. Rodgers, O.D. Underwood, J.D. Madison, K.R. Ford, S.R. Whetten, D.J. Dagel, and J.E. Bishop, Comput. Mech. 61(5), 559 (2018).

27. T.M. Rodgers, J.A. Mitchell, and V. Tikare, Modell. Simul. Mater. Sci. Eng. 25(6), 064006 (2017).

28. T.M. Rodgers, J.D. Madison, V. Tikare, and M.C. Maguire, JOM 68(5), 1419 (2016)

29. T.M. Rodgers, J.E. Bishop, and J.D. Madison, Modell. Simul. Mater. Sci. Eng. 26(5), 055010 (2018).

30. F. Bachmann, R. Hielscher, and H. Schaeben, Texture Analysis with MTEX-Free and Open Source Software Toolbox, Solid State Phenomena, vol. 160 (Trans Tech Publications, Zurich, 2010).

31. D. Peirce, R. Asaro, and A. Needleman, Acta Metall. 30(6), 1087 (1982).

32. S.R. Kalidindi, C.A. Bronkhorst, and L. Anand, J. Mech. Phys. Solids 40, 537 (1992).

33. H. Lim, J.D. Carroll, C.C. Battaile, B.L. Boyce, and C.R. Weinberger, Int. J. Mech. Sci. 92, 98 (2015).

34. H. Lim, H.J. Bong, S.R. Chen, T.M. Rodgers, C.C. Battaile, and J.M.D. Lane, Mater. Sci. Eng. A 730, 50-56 (2018).

35. J.W. Hutchinson, Proc. R. Soc. Lond. A 348, 101 (1976).

36. S.B. Brown, K.H. Kim, and L. Anand, Int. J. Plast. 5, 95 (1989).

37. V. Popovich, E. Borisov, A. Popovich, V. Sufiiarov, D. Masaylo, and L. Alzina, Mater. Des. 114, 441 (2017).

38. T.R. Smith, J.D. Sugar, C.S. Marchi, and J.M. Schoenung, in ASME 2017 Pressure Vessels and Piping Conference (American Society of Mechanical Engineers, 2017), pp. V06AT06A020-V06AT06A020.

39. J. Liu, W. Xiong, A. Behera, S. Thompson, and A.C. To, Int J. Solids Struct. 112, 35 (2017).

40. Y.M. Wang, T. Voisin, J.T. McKeown, J. Ye, N.P. Calta, Z Li, Z. Zeng, Y. Zhang, W. Chen, T.T. Roehling, R.T. Ott, M.K. Santala, P.J. Depond, M.J. Matthews, A.V. Hamza, and T. Zhu, Nat. Mater. 17(1), 63 (2017).

41. L. Liu, Q. Ding, Y. Zhong, J. Zou, J. Wu, Y.L. Chiu, J. Li, Z Zhang, Q. Yu, and Z. Shen, Mater. Today 21(4), 354 (2018).

42. R. Cunningham, S.P. Narra, C. Montgomery, J.L. Beuth and A.D. Rollett, JOM 69(3), 479 (2017).

43. J.D. Madison, O.D. Underwood, L.P. Swiler, B.L. Boyce, B.H. Jared, J.M. Rodelas, and B.C. Salzbrenner, AIP Conf. Proc. 1949(1), 020009 (2018).

44. B.L. Boyce, B.C. Salzbrenner, J.M. Rodelas, L.P. Swiler, J.D. Madison, B.H. Jared, and Y.L. Shen, Adv. Eng. Mater. 67, 1700102 (2017)

45. B.C. Salzbrenner, J.M. Rodelas, J.D. Madison, B.H. Jared L.P. Swiler, Y.L. Shen, and B.L. Boyce, J. Mater. Process. Technol. 241, 1 (2017).

Publisher's Note Springer Nature remains neutral with regard to jurisdictional claims in published maps and institutional affiliations. 